Mon. Not. R. Astron. Soc. 000, 000-000 (0000) Printed 28 October $2018 \quad$ (MN LATEX style file v1.4)

\title{
Do LINER 2 galaxies harbour low-luminosity Active Galactic Nuclei?
}

\author{
T. P. Roberts, N. J. Schurch and R. S. Warwick \\ Department of Physics and Astronomy, University of Leicester, University Road, Leicester, LE1 7RH
}

\begin{abstract}
We use ROSAT HRI spatial data and ASCA spectral measurements for a sample of seven nearby, early type spiral galaxies, to address the question of whether a lowluminosity Active Galactic Nucleus (LLAGN) is present in galaxies that have a LINER 2 classification. The brightest discrete X-ray source in the ROSAT HRI observations is invariably found to be positionally coincident with the optical galactic nucleus, and in most cases its flux dominates the $\mathrm{X}$-ray emission from the central region of the galaxy. All seven galaxies have X-ray spectra consistent with a two-component, soft thermal plus hard power-law, spectral form. If we exclude the two galaxies with relatively hard X-ray spectra, NGC 3628 and NGC 4594, for which there is supporting evidence for a LLAGN (or alternatively in the case of NGC 3628 a dominant ultraluminous X-ray binary), then the remaining galaxies show surprisingly similar X-ray spectral properties. Specifically the flux ratio $F_{X}(0.5-1) / F_{X}(2-5)$, which measures the relative strengths of the thermal and non-thermal emission components, shows little scatter about a mean of 0.66 , a value very similar to that measured in the classic starburst galaxy NGC 253. Since there is no obvious reason why the luminosity of the hard power-law continuum emanating from a putative LLAGN should be very closely correlated with the thermal emission of the surrounding region, this suggests that that the broad-band (0.5-5 keV) X-ray emission from these LINER 2 galaxies may originate in a common set of processes probably associated with the starburst phenomenon. Conversely, it appears that in many, perhaps the majority, of LINER 2 galaxies, the nuclear X-ray luminosity does not derive directly from the presence of a LLAGN.
\end{abstract}

Key words: galaxies:active - galaxies:nuclei - X-rays:galaxies

\section{INTRODUCTION}

The widespread nature of the low-ionization nuclear emission-line region (LINER) phenomenon (Heckman 1980) has recently been underlined by Ho, Filippenko \& Sargent (1997, hereafter HFS), who find that galaxies containing LINERs comprise $\sim 33 \%$ of the local bright galaxy population, outnumbering low-luminosity Seyfert nuclei by a ratio of $3: 1$. The excitation mechanisms giving rise to the characteristic optical spectra of LINERS remain the subject of considerable debate. Plausible models include photoionization by a non-stellar continuum source, namely a low-luminosity active galactic nucleus (LLAGN), collisional ionization by fast shocks (Heckman 1980) and photo-ionization by the UV radiation generated by clusters of hot, young stars (Terlevich \& Melnick 1985, Shields 1992).

The detection by HFS of broad ( $\sim$ few thousand $\mathrm{km}$ $\mathrm{s}^{-1}$ ) permitted lines in the optical spectra of $\sim 20 \%$ of LINERs points firmly to the presence of a LLAGN in a subset of the population. This also prompted HFS to introduce a "type 1" and "type 2" classification for LINERS, paralleling the established nomenclature for Seyfert galaxies. In light of the fact that many nearby galaxies appear to contain a central supermassive black hole (see Lawrence 1999 for a review), it is a reasonable conjecture that the majority of LINERs (i.e., all type 1 plus a significant fraction of type 2 systems) may be powered by a LLAGN. Such a conclusion has consequences for a range of astrophysical issues. It would, for example, imply that LINERs constitute the lowluminosity end of the local AGN population with ramifications for the evolution of the AGN luminosity function and the history of supermassive black hole formation in galaxies.

$\mathrm{X}$-ray observations can provide important clues as to the physical processes responsible for LINERs. Ideally, with good spatial resolution, extended nebulosity can be resolved to reveal the presence (or absence) of a compact X-ray source identifiable as the LINER nucleus. With moderate spectral resolution a non-thermal AGN-like spectrum is then readily distinguished from a thermal (hot gas) spectrum. Even when the spatial resolution fails to separate all the components, 
spectral modelling may allow the various contributors to the composite spectrum to be distinguished. Furthermore, since X-ray observations can be used to detect a LLAGN which is inconspicuous in the optical band (either due to its low contrast against the stellar backdrop or the effects of nuclear obscuration), X-ray measurements provide an effective way of testing the hypothesis that the majority of LINER 2's are accretion-powered systems.

In the present paper we focus our attention on a small sample of nearby galaxies classified by HFS as having either a pure LINER 2 optical spectrum or a composite LINER 2/HII spectrum. HFS refer to the latter as transition LINERs. For all the sources in our sample, X-ray spectral information is available from $A S C A$ and high spatial resolution $\mathrm{X}$-ray imaging from $R O S A T$.

\section{THE LINER 2 SAMPLE}

The LINER 2 galaxies studied in this paper were selected primarily on the basis of the availability of appropiate $A S C A$ and ROSAT High Resolution Imager (HRI) datasets. As a first step, the positions of the 133 galaxies classified as LINER 2 (L2) or Transition 2 (T2) objects by HFS were cross-correlated with the pointing positions of the ASCA observations comprising the ASCAPUBLIC database available at the Leicester Database and Archive Service (LEDAS) This lead to a preliminary list of $31 \mathrm{~L} 2 / \mathrm{T} 2$ galaxies. This sample was further reduced by eliminating all the galaxies that were not the target of the $A S C A$ observations. Also at this stage elliptical and lenticular galaxies were excluded from the sample, due to concern that X-ray emission associated with a hot ISM component in these galaxies might confuse the search for nuclear X-ray activity. Discarding the only galaxy of the 8 remaining galaxies that has not been observed with the ROSAT HRI instrument, results in a small homogeneous sample of 7 early-type spiral galaxies. Basic details of these seven galaxies, taken from HFS, are given in Table 11. Also the specific $A S C A$ and ROSAT HRI datasets used in the present analysis are listed in Table 2. Information on the LINER nucleus and the X-ray properties of each galaxy is summarised in the Appendix.

\section{THE $R O S A T$ HRI OBSERVATIONS}

The ROSAT HRI observations have been used to examine the X-ray morphology of each of the galaxies in our sample. The HRI measurements cover a soft $(0.1-2.4 \mathrm{keV})$ energy range and lack the sensitivity to highly obscured $\left(N_{H}>10^{22.5} \mathrm{~cm}^{-2}\right)$ sources afforded by the harder $(0.6-$ $8 \mathrm{keV}$ ) effective bandpass of $A S C A$. However, the former has a massive advantage over the latter in terms of spatial resolution with a point spread function FWHM of $\sim 5^{\prime \prime} \mathrm{com}-$ pared to $\sim 90^{\prime \prime}$. Although some previous galaxy studies (e.g. Ptak et al. 1997; Terashima et al. 2000a) have attempted to utilise the imaging capabilities of $A S C A$, the relatively low count rates combined with the inherent poor spatial resolution dissuades us from adopting this approach in the present

\footnotetext{
^ We also include a proprietary observation of NGC 3627 in our sample.
}

study. Here we focus solely on the spatial information available from the ROSAT HRI observations.

We have analysed the deepest ROSAT HRI observation available for each galaxy (see Table 2). The spatial analysis was performed in two stages. Firstly, the raw HRI images were smoothed using a Gaussian mask with FWHM of $15^{\prime \prime}$, and the corresponding X-ray contour maps were overlaid onto Digitised Palomar Sky Survey images as shown in Figure 1. Secondly, a discrete source search was undertaken using the PSS (Point Source Search) algorithm, as described in Roberts \& Warwick (2000). All the X-ray sources having a statistical significance of $5 \sigma$ or greater lying within the $\mathrm{D}_{25}$ isophotal ellipse of each galaxy were recorded.

\section{Insert Figure 1 here.}

The most striking feature of the images in Figure 1 is that the X-ray emission coincident with the each galactic disk appears to peak at the position of the nucleus of the galaxy. This result is verified by the PSS, which detects a point-like source within $\sim 30^{\prime \prime}$ of the optical nuclear position in every image. These apparently ubiquitous nuclear X-ray sources are, without exception, the most luminous pointlike X-ray source associated with each galaxy. The observed HRI count rate of the point-like nuclear component, $C_{n u c}$, and the corresponding (Galactic absorption-corrected) Xray luminosity, $\mathrm{L}_{\mathrm{X}}$, is given in Table 3 . Here we derive $\mathrm{X}$-ray luminosity (in the $0.1-2.4 \mathrm{keV}$ band) using the same spectral assumptions as employed by Roberts \& Warwick (2000).

Table 3 also lists the fraction, $f_{n u c}$, of the counts measured in the HRI image within a radius of $4^{\prime}$ of the nucleus, which are attributable to the point-like nuclear X-ray source. (A radius of $4^{\prime}$ was chosen to match the source extraction region used in the $A S C A$ analysis.) Clearly $f_{n u c}$ is an indicator of how dominant the nuclear X-ray source is in comparison to the other contributors (both extended and point-like) to the central X-ray emission from each galaxy. For the majority of the sources the nuclear point component represents a substantial fraction of the total central X-ray emission, with NGC 3627 at $~ 15 \%$ having the lowest fractional nuclear contribution.

To the best of our knowledge the ROSAT HRI observations of NGC 3627 and NGC 7217 are previously unpublished. NGC 3627 is particularly interesting since its nuclear emission is relatively weak (c.f. Table 3) with the bulk of the central soft X-ray emission originating in an irregular diffuse component with an angular scale of $\sim 3^{\prime}$. The northern tip of this extended component overlaps with the position of the recent supernovae SN1989B. Two extra-nuclear point-sources are detected (to the east of nucleus) with Xray luminosities (corrected for Galactic absorption) of 4.5 and $9 \times 10^{38} \mathrm{erg} \mathrm{s}^{-1}$ respectively. These may be two further examples of "super-luminous objects", a heterogeneous class of very luminous non-nuclear X-ray sources which have been observed in a number of nearby galaxies (Roberts \& Warwick 2000). NGC 3627 is listed as an AGN candidate by Filho, Barthel \& Ho (2000) on the basis of their VLA observations. This together with the ambiguous T2/S2 spectral classification assigned by HFS might be explained if NGC 3627 hosts a Seyfert 2-like nucleus, which is heavy absorbed along the line of sight. In this scenario the observed X-ray 
Table 1. Properties of the galaxies in the sample.

$\begin{array}{lccccc}\text { Name } & \text { Nuclear class } & T & \text { Hubble type } & \text { Distance (Mpc) } & \text { M }_{\text {B,bulge }} \\ \text { NGC 3627 } & \text { T2/S2 } & 3 & \text { SAB(s)b } & 6.6 & -18.43 \\ \text { NGC 3628 } & \text { T2 } & 3 & \text { SAb pec spin } & 7.7 & -18.58 \\ \text { NGC 4321 } & \text { T2 } & 4 & \text { SAB(s)bc } & 16.8 & -19.18 \\ \text { NGC 4569 } & \text { T2 } & 2 & \text { SAB(rs)ab } & 16.8 & -20.11 \\ \text { NGC 4594 } & \text { L2 } & \text { 1 } & \text { SA(s)a spin } & 20.0 & -22.11 \\ \text { NGC 4736 } & \text { L2 } & 2 & \text { (R)SA(r)ab } & 4.3 & -18.19 \\ \text { NGC 7217 } & \text { L2 } & 2 & \text { (R)SA(r)ab } & 16.0 & -19.26\end{array}$

Table 2. The ROSAT and ASCA datasets used in the present analysis.

\begin{tabular}{lccccc} 
Galaxy & \multicolumn{2}{c}{ ROSAT data } & \multicolumn{3}{c}{$A S C A$ data } \\
& ID & Exposure (s) & ID & SIS exp.(s) & GIS exp.(s) \\
NGC 3627 & rh600836a01 & 9456 & 67031000 & 19345 & 24688 \\
NGC 3628 & rh700009n00 & 13574 & 61015000 & 23904 & 26912 \\
NGC 4321 & rh600731n00 & 42797 & 55044000 & 27296 & 30336 \\
NGC 4569 & rh600603a01 & 21858 & 65012000 & 19520 & 21728 \\
& & & 65012010 & 19424 & 20960 \\
NGC 4594 & rh600044a02 & 15850 & 61014000 & 19552 & 20864 \\
NGC 4736 & rh600678n00 & 112910 & 63020000 & 28032 & 32736 \\
NGC 7217 & rh702933a01 & 10791 & 63009000 & 79552 & 86624
\end{tabular}

luminosity could arise as nuclear continuum flux which is electron scattered into the line of sight by highly photoionized gas extending above (and below) the obscuring matter.

\section{THE ASCA OBSERVATIONS}

The details of the ASCA SIS and GIS datasets used in the present analysis are given in Table 2. Bright-2 mode data were available for all the sources with the exception of NGC 3628 for which only Bright mode data were available. The data were analysed using standard procedures and datascreening criteria. Images from each $A S C A$ instrument were examined and raw spectra and light curves extracted, using a circular aperture of radius $4^{\prime}$ centred on the source position. Complementary off-source (i.e. background) information was also obtained for each dataset, and subtracted in the spectral fitting process. The net source count rates were in the range $0.009-0.85$ count $\mathrm{s}^{-1}$ for the SIS and $0.006-0.55$ count $\mathrm{s}^{-1}$ for the GIS. In each case the analysis concentrated on the temporal and spectral properties of the target galaxy.

In the case of the temporal analysis, the data from each $A S C A$ telescope were first background subtracted and then binned on a timescale of a quarter of an $A S C A$ orbit (1440 seconds). The resulting light curves showed no evidence for significant variations on any sampled timescale. In the case of NGC 4569 two separate observations were available but again no significant variations were apparent between the two datasets.

The response matrices and ancillary response files for the spectral investigation of the SIS data were created using the standard $A S C A$ FTOOLS. The most recent standard response matrices and ancillary response files were used for the GIS instrument analysis. The observation-averaged and background-subtracted spectral data were binned to a minimum of 20 counts per spectral channel over the range 0.6 $10 \mathrm{keV}$ for the SIS and $0.8-10 \mathrm{keV}$ for the GIS instruments.
The spectral modelling was carried out using the XSPEC V10.0 software package. Data from all four $A S C A$ instruments were fit simultaneously but with the relative model normalisation allowed to vary freely. The quoted errors on the derived best-fitting model are based on a $\Delta \chi^{2}=2.71$ criterion, that is at a 90 per cent confidence level for one interesting parameter.

The data for each source were initially fit with a model comprising a power-law continuum of photon spectral index, $\Gamma$, with low-energy absorption arising in a line-of-sight hydrogen column density, $N_{H}$. In terms of the minimum $\chi^{2}$, this simple model provided quite a reasonable description of observed spectra for four of the sources (NGC 3627, NGC 3628, NGC 4321, NGC 7217) with the measured values of $\Gamma$ ranging from $1.4-2.3$ and low values of column density $\left(N_{H}<2 \times 10^{21}\right)$, consistent with the Galactic value in three of the four sources.

Ptak et al. (1999) have shown that the observed Xray spectra of low-luminosity galactic nuclei, whether driven by accretion or starburst processes, can often be fitted by a "standard spectral model". This model consists of soft X-ray emission attributed to a Raymond-Smith plasma $\left(T_{R S} \lesssim 10^{7} \mathrm{~K}\right)$, which contributes mostly below $\sim 2 \mathrm{keV}$, plus a harder emission component which can be modelled either as a power-law $(\Gamma \approx 1.7)$ or a thermal bremsstrahlung $(k T \approx 5 \mathrm{keV})$ continuum. The soft Raymond-Smith component generally shows little evidence for absorption over and above the line-of-sight Galactic column density whereas the hard component often exhibits some additional intrinsic column density (typically $N_{H, \text { int }} \sim 10^{22} \mathrm{~cm}^{-2}$ ). In addition the abundances inferred for the Raymond-Smith plasma are generally significantly sub-solar, although this may be more indicative of a complex multi-temperature plasma than a true under-representation of elements such as iron and oxygen.

Given the relatively low count rates, and hence restricted signal-to-noise, of the X-ray spectra for sources in 

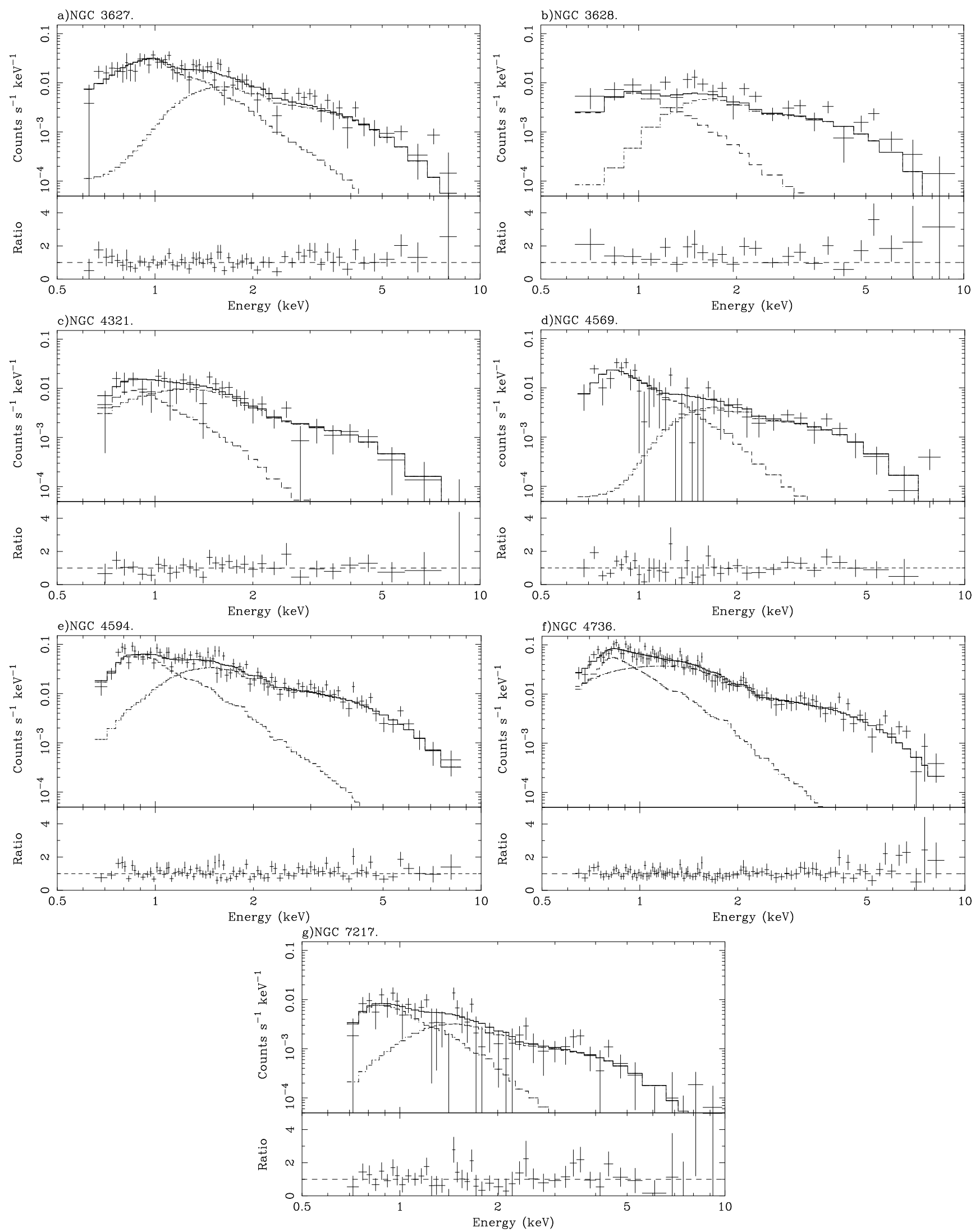

Figure 2. The $A S C A$ count rate spectra of the seven LINER 2 galaxies. For clarity only the SIS 0 data are shown. In each case the lower panel shows the ratio of the observed spectrum to the best fitting model. 
Table 3. Nuclear X-ray sources detected in the HRI images.

\begin{tabular}{lcrc} 
Source & $C_{\text {nuc }}{ }^{a}$ & $\log L_{X}{ }^{b}$ & \multicolumn{1}{c}{$f_{n u c}$} \\
NGC 3627 & $3.9 \pm 0.8$ & 39.00 & $0.15_{-0.04}^{+0.05}$ \\
NGC 3628 & $12.3 \pm 1.0$ & 39.62 & $1.00_{-0.08}^{+0}$ \\
NGC 4321 & $8.2 \pm 0.5$ & 40.13 & $0.42_{-0.05}^{+0.06}$ \\
NGC 4569 & $5.1 \pm 0.6$ & 39.93 & $0.51_{-0.11}^{+0.14}$ \\
NGC 4594 & $17.3 \pm 1.2$ & 40.65 & $0.51 \pm 0.07$ \\
NGC 4736 & $50.5 \pm 0.7$ & 39.69 & $0.62 \pm 0.02$ \\
NGC 7217 & $2.3 \pm 0.6$ & 39.71 & $0.39_{-0.16}^{+0.25}$
\end{tabular}

\footnotetext{
${ }^{a}$ In units of $10^{-3}$ HRI count $\mathrm{s}^{-1}$

${ }^{b} \mathrm{In} \operatorname{erg~s}^{-1}(0.1-2.4 \mathrm{keV})$
}

Table 4. Results of the spectral fitting of the $A S C A$ observations.

$\begin{array}{lccccccc}\text { Source } & k T(\mathrm{keV}) & \mathrm{N}_{H, G a l}{ }^{a} & \mathrm{~N}_{H, I n t}{ }^{b} & \Gamma & \chi^{2} / \text { d.o.f. } & \Delta \chi^{2} & \log L_{X}{ }^{c} \\ \text { NGC 3627 } & 0.88_{-0.07}^{+0.02} & 2.4 & 1.7_{-0.63}^{+0.82} & 2.6_{-0.37}^{+0.38} & 196.6 / 202 & 16.9 & 39.81 \\ \text { NGC 3628 } & 0.85_{-0.30}^{+\infty} & 2.2 & 1.5_{-0.70}^{+0.79} & 2.0_{-0.38}^{+0.40} & 89.6 / 85 & 11.0 & 39.85 \\ \text { NGC 4321 } & 0.67_{-0.67}^{+0.66} & 2.4 & 0.2_{-0.20}^{+1.54} & 1.9_{-0.48}^{+0.56} & 77.9 / 91 & 1.6 & 40.33 \\ \text { NGC 4569 } & 0.65_{-0.10}^{+0.09} & 2.5 & 2.3_{-0.73}^{+1.27} & 2.6_{-0.53}^{+0.61} & 155.2 / 133 & 48.5 & 40.34 \\ \text { NGC 4594 } & 0.76_{-0.17}^{+0.07} & 3.8 & 0.8_{-0.34}^{+0.44} & 1.96_{-0.14}^{+0.14} & 272 / 239 & 30.6 & 41.19 \\ \text { NGC 4736 } & 0.64_{-0.07}^{+0.05} & 1.44 & 0.03_{-0.30}^{+0.12} & 1.60_{-0.08}^{+0.15} & 277.6 / 281 & 201.3 & 39.53 \\ \text { NGC 7217 } & 0.70_{-0.23}^{+0.13} & 0.11 & 0.64_{-0.64}^{+0.70} & 1.8_{-0.38}^{+0.44} & 146.7 / 168 & 13.0 & 40.10\end{array}$

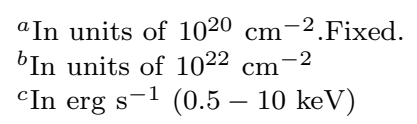

our current sample, we have applied some additional restrictions in our usage of the above "standard spectral model". Thus the Raymond-Smith component is assumed (i) to be subject only to absorption by the line of sight Galactic column density, (ii) to have temperature $k T_{R S}$ in a restricted range of $0.6-0.9 \mathrm{keV}$ (corresponding to the peak in the temperature distribution measured by Ptak et al. 1999, see their Fig.4) and (iii) to have a metal abundance fixed at 0.1 times the solar value. Table 1 details the results from the fitting of this standard spectral model. This standard model provides an excellent match to the observed spectra and in all cases bar one, it provides a very significant improvement in the minimum $\chi^{2}$ in compared to the simple absorbed power-law model (i.e. $\Delta \chi^{2}$ in Table (1). Table 4 also lists the observed 0.5-10 keV X-ray luminosity for each source (corrected for Galactic absorption). Fig. 2 shows the $A S C A$ count rate spectra and the corresponding best fitting model.

As a final step we have attempted to fit all the sources with an iron $\mathrm{K}_{\alpha}$ line. This feature is seen in several LLAGN, though it is a matter of debate whether it is indicative of $\mathrm{X}$-ray reprocessing in matter surrounding an active nucleus, as is the case in more luminous AGN (see Terashima et al. $2000 \mathrm{~b}$, and references therein). Given the restricted signal to noise we first add a narrow Gaussian line component to the best-fitting model at a fixed energy. Table 5 summarises the limits on the line equivalent width of lines placed at energies of $6.4 \mathrm{keV}$ (corresponding to low ionization states for iron) and $6.7 \mathrm{keV}$ (helium-like iron). When we then set the line energy as a free parameter in the range $6.4-6.96 \mathrm{keV}$, there were $\mathrm{Fe} \mathrm{K}_{\alpha}$ line detections in only two sources (NGC 4736 and NGC 7217). However, both detections were marginal, most particularly in the case of NGC 4736 (see Table 5)

\section{DISCUSSION}

Our objective in focussing this analysis on a relatively homogeneous, albeit small, sample of early type spiral galaxies has been to investigate whether there are any properties that the sample galaxies have in common, which may be linked to their LINER 2 classification. In principle, if such a link could be established this might provide some clue as to whether LINER 2 characteristics are derived from the presence of a LLAGN or by some alternative shared mechanism.

\subsection{Comparing ROSAT HRI and $A S C A$ observations}

As noted previously, the ROSAT HRI images reveal the presence of a point-like X-ray source coincident with the optical nucleus in all seven galaxies. The inferred soft X-ray luminosities of these "nuclear" X-ray sources (hereafter NXS) ranges from $1.0-45 \times 10^{39} \mathrm{erg} \mathrm{s}^{-1}$ (Table 3). In most of the galaxies the NXS contributes $\sim 50 \%$ or more of the central galactic soft X-ray emission. Though the presence of a dominant NXS indicates a strong soft X-ray emission peak coincident with the nucleus it is not, in isolation, unambiguous evidence for a LLAGN. The $\sim 5^{\prime \prime}$ spatial resolution of the HRI translates to a linear scale of $100-500$ pc for the sample galaxies, which covers the inner bulge component of 
Table 5. $90 \%$ upper limits to the line equivalent width for various iron $\mathrm{K} \alpha$ configurations.

$\begin{array}{lcccc}\text { Source } & \begin{array}{c}\text { 6.4 keV line } \\ \text { Eq.Width (eV) }\end{array} & \begin{array}{c}6.7 \mathrm{keV} \text { line } \\ \text { Eq.Width (eV) }\end{array} & \begin{array}{c}\text { Free line } \\ \text { Energy }(\mathrm{keV})\end{array} & \begin{array}{c}\text { Free line } \\ \text { Eq. Width }(\mathrm{eV})\end{array} \\ \text { NGC 3627 } & 467 & 710 & - & - \\ \text { NGC 3628 } & 694 & 859 & - & - \\ \text { NGC 4321 } & 670 & 804 & - & - \\ \text { NGC 4569 } & 655 & 486 & - & - \\ \text { NGC 4594 } & 205 & 316 & - & - \\ \text { NGC 4736 } & 350 & 604 & 6.72^{a} & 597 \\ \text { NGC 7217 } & 606 & 1320 & 6.94_{-0.64}^{+0.26} & 1688\end{array}$

${ }^{a}$ Line energy unconstrained at the $90 \%$ level

each galaxy and will encompass any nuclear and/or circumnuclear starburst regions in addition to any putative active nucleus. Studies of normal spiral and starburst galaxies have demonstrated that X-ray luminosities of up to $10^{40} \mathrm{erg} \mathrm{s}^{-1}$ can be generated through the summed emission of the underlying galactic X-ray source populations (high and low mass $\mathrm{X}$-ray binaries, recent supernovae and supernova remnants) and energetic diffuse processes (Fabbiano 1989). For most of our sample this can provide an alternative explanation for the observed nuclear X-ray emission. Clearly further constraints are required based on either the temporal or spectral properties of the NXS revealed by the ROSAT observations.

The X-ray spectra measured by $A S C A$ for this set of galaxies show remarkable similarities as evidenced by the fact that our "standard", thermal plus absorbed powerlaw, spectral model provides a good fit in all seven cases. Fig. 2 illustrates the relative contributions of the thermal and power-law components to the observed count rate spectra (as derived from the spectral fitting). Interestingly, the cross-over between the two spectral components, for this set of sources, occurs within a very narrow energy range, namely $0.9-1.5 \mathrm{keV}$. Since the spectral model implies that the measured flux in the $0.5-1 \mathrm{keV}$ band (corrected for Galactic absorption) is largely attributable to thermal emission, whereas that in the $2-5 \mathrm{keV}$ band is attributable to the power-law component, it follows that the ratio $F_{X}(0.5-1) / F_{X}(2-5)$ provides a measure of the relative strength of the two components. This ratio is tabulated in Table for each galaxy. Excluding NGC 3628 and NGC 4594 , for which there is good existing evidence for the presence of a LLAGN or, alternately in the case of NGC 3628, an ultra-luminous binary dominant in the X-ray regime (see the notes in the Appendix), the five remaining sources have a mean value for this ratio of 0.66 with a standard deviation of only 0.10 . This commonality of spectral form argues strongly that the $0.5-5 \mathrm{keV} \mathrm{X}$-ray flux derives from the same emission process (or a linked set of processes) in these five galaxies. This is a rather powerful argument against the LLAGN hypothesis since there is no obvious reason why the soft thermal emission should be so closely correlated with the level of the hard power-law continuum emanating from a LLAGN. In terms of the above spectral ratio, the X-ray spectra of NGC 3628 and NGC 4594 are, respectively, factors of 3.7 and 1.7 harder than the average for the other five galaxies; it is plausible that this is due to the contribution of an underlying LLAGN in these two galaxies.

It is notable that when $F_{X}(0.5-1) / F_{X}(2-5)$ is derived for the classic starburst galaxy NGC 253, based upon the $A S C A$ data fits of Ptak et al. (1999), an identical value of 0.66 is obtained. Recent analysis of the XMM-Newton PV observation of this galaxy (Pietsch et al. 2001) has shown its X-ray emission to be dominated by thermal (i.e. stellar) processes, with no evidence at all for a LLAGN component. It appears, then, that the $F_{X}(0.5-1) / F_{X}(2-5)$ of $\sim 0.66$ is readily explained by stellar processes alone. We note that this result cannot be corroborated by analysis of the other classic starburst galaxy, M82, since on some occasions the Xray emission of this galaxy is dominated by a single, variable, luminous X-ray source (Matsumoto et al. 2001).

The $A S C A$ measurements sample a relatively large area of each galaxy (a $4^{\prime}$ radius region centred on the nucleus) with a harder spectral response than that of the ROSAT HRI. As a result it is not straightforward to relate the $A S C A$ spectral measurements to the properties of the NXS identified in the ROSAT images. One approach is to assume that the absorbed power-law emission originates solely in the NXS. Using appropriate HRI response files in XSPEC, we have derived the expected ROSAT HRI count rate for the power-law component based on the spectral parameters and normalisations obtained from the $A S C A$ measurements. In all cases the predicted count rate is less than that actually observed from the NXS. Variability might, in principle, give rise to some disparity between the $A S C A$ and ROSAT measurements, but only in the case of NGC 3628 is there explicit evidence for such behaviour (see the notes in the Appendix). This bias towards the under-prediction of the NXS flux is perhaps explained if at least some of the thermal emission modelled in $A S C A$ also originates from the compact nuclear source.

An alternative viewpoint would be to suggest that the hard power-law emission does not originate solely in the nucleus. This would certainly appear to be consistent with the results of Terashima et al.(2000a), who report that the hard X-ray emission associated with several nearby LINER 2 nuclei is extended on scales of several kpc. In this case, the soft X-ray NXS could be almost entirely dominated by the thermal component. This, again, supports the assertion that the LINER 2 nuclei need not harbour LLAGN. Unfortunately further quantitative analysis of the two scenarios outlined above cannot be justified given the mis-match of the $A S C A$ and ROSAT HRI bandpasses. For example, ROSAT PSPC and HRI measurements indicate the presence of a very soft thermal $(\sim 0.1 \mathrm{keV})$ component in NGC 4736 which is not evident in the $A S C A$ spectra (Roberts, Warwick 
\& Ohashi 1999). Clearly much more definitive analysis will be possible with observations combining good spectral and excellent spatial resolution, such as those from $C H A N D R A$.

\subsection{Luminosity ratio diagnostics}

The ratio of the X-ray luminosity to the luminosity in other wavebands can be a very useful diagnostic of the underlying emission processes occurring within a galaxy. It has long been suspected that the hard component present in the Xray spectra of normal (i.e. non-active) early-type galaxies is the signature of a ubiquitous population of low-mass X-ray binaries (LMXBs), and that the X-ray luminosity of this component is well correlated with the blue luminosity of the galaxy (e.g. Canizares et al. 1987; Matsushita et al. 1994). Iyomoto et al. (1998) have recently used this relationship to demonstrate that LLAGN are probably present in the elliptical galaxies NGC 3065 and NGC 4203, by showing that the ratio of the $2-10 \mathrm{keV}$ X-ray luminosity contained in a hard power-law component, to the galactic blue luminosity, is two orders of magnitude greater for these galaxies than in normal ellipticals (c.f. Fig. 5 of Iyomoto et al. 1998). The same test can be applied to the present LINER 2 sample using the blue luminosity of their galaxy bulges. The ratio $L_{X(2-10, \mathrm{PL})} / L_{B, \text { bulge }}$ (hereafter $\left.L_{X} / L_{B}\right)$ is listed in Table 6 . It shows remarkably little scatter throughout the sample, especially when we consider that the inherent range in the bulge blue luminosity is $\sim 1.6-60 \times 10^{9} L_{\odot}$, i.e. a factor of $\sim$ 40. For the early-type galaxies in Iyomoto et al., typically log $L_{X} / L_{B} \approx-4$.1. Similarly Irwin \& Bregman (1999) obtain a value $\log L_{X} / L_{B}=-4.26$ for the bulge component of M31. The implication is that the LINER 2s studied here have an observed X-ray luminosity which is a factor $4-10$ higher than the predicted LMXB contribution. The excess $\mathrm{X}$-ray emission may be directly attributable to the presence of a LLAGN, and indeed the $\log L_{X} / L_{B}$ values in Table 6 are similar to those found for bona fide LLAGN in spiral galaxies (Iyomoto et al. 1998). However, given their later morphological type in comparison to the galaxies studied by Iyomoto et al. (1998), an equally plausible explanation is that our LINER 2 sample have nuclei hosting a more energetic (and perhaps younger) X-ray binary population. The absence of rapid (or, in fact, any) temporal variations in the hard X-ray emission from our sample is consistent with the hard flux originating in an unresolved X-ray binary population.

If the dominant contribution to the X-ray luminosity in our LINER 2 sample is attributable to processes associated with an underlying young stellar population, then we might expect to see the X-ray luminosity associated with the hot gas component scale with other measures of star-formation activity. Terashima et al. (2000a) test for this by comparing the intrinsic $0.5-4 \mathrm{keV}$ X-ray luminosity of the hot thermal component in spectra of several LINER 2 galaxies with the far-infrared luminosity of the host galaxy, i.e. by examining the ratio $L_{X(0.5-4, \mathrm{RS})} / L_{F I R}$ (hereafter $\left.L_{X} / L_{F I R}\right)$. Known starburst galaxies have $\log L_{X} / L_{F I R} \approx-4 \pm 1$ and, in terms of this ratio, Terashima et al. (2000a) find that their LINER 2 galaxies have somewhat similar characteristics. The same comment also applies to the present sample (see Table 6, where we used $L_{F I R}$ values published in HFS). The exception to this result is NGC 4594 which has a notably higher
X-ray to FIR ratio. This anomaly may be due to the presence of a hot ISM associated with the dominant massive bulge component in NGC 4594. We can conclude that, aside from NGC 4594, the X-ray luminosity residing in the thermal component is at a level consistent with a starburst origin. Furthermore, the constancy of spectral form noted in the previous section would imply that the hard power-law component also has a starburst connection and that this is the origin of the apparent excess of hard flux noted above.

Terashima et al. (2000a) also discussed the $\mathrm{H} \alpha$ ionising photon budget for low luminosity Seyfert galaxies and for LINERS. Specifically they investigate whether the underlying UV to X-ray continuum can produce the observed $\mathrm{H} \alpha$ flux, or whether additional ionising sources are required. Following a number of assumptions, they conclude that log $L_{X} / L_{H \alpha} \geq 1.4$ is required to explain the $\mathrm{H} \alpha$ luminosity solely in terms of photoionization from a LLAGN. The sample of LINER 2 s considered by Terashima et al.(2000a) all have $\log L_{X} / L_{H \alpha} \leq 0.6$ implying either the primary ionisation source is not a LLAGN or that the line of sight to the LLAGN is heavy obscured in X-rays and possibly Compton thick. Derived values for the $L_{X} / L_{H \alpha}$ ratio for the present sample of LINER $2 \mathrm{~s}$ are given in Table (where $L_{X}=L_{X(2-10, \mathrm{PL})}$ and $L_{H \alpha}$ is taken from HFS). In the case of NGC 3628, the hard X-ray to $\mathrm{H} \alpha$ luminosity ratio far exceeds the $\mathrm{H} \alpha$ ionisation requirement, consistent with presence of a LLAGN. At the other extreme NGC 4569 and NGC 7217 would appear to be under-luminous in X-rays in relation to their observed $\mathrm{H} \alpha$ flux. The four remaining galaxies in our sample have $\log L_{X} / L_{H \alpha}=1-1.5$, a somewhat indeterminate level in terms of identifying the presence or absence of a LLAGN.

\section{CONCLUSION}

There is rather mixed evidence concerning the presence of a LLAGN in the seven LINER 2 galaxies which comprise the present sample. NGC 3628 is a highly variable X-ray source (Dahlem, Heckman \& Fabbiano 1995), has an X-ray spectrum that is dominated by a hard component and has a high $L_{X} / L_{H \alpha}$ ratio, which may possibly originate in a LLAGN, though it is also possible that its X-ray emission is dominated by an ultra-luminous X-ray binary system. NGC 4594 also shows some anomalous X-ray properties but displays the best observational evidence for a LLAGN, which is provided by the recent discovery of broad wings on its nuclear emission lines (Kormendy et al. 1996) plus the incontrovertible case for a $10^{9} \mathrm{M}_{\odot}$ black hole in this galaxy (Kormendy 1988). At the other extreme, both NGC 4569 and NGC 7217 appear to be LINER 2 systems driven predominantly by stellar processes, one manifestation of which is their low $L_{X} / L_{H \alpha}$ ratio. For the three remaining sources, NGC 3627, NGC 4321 and NGC 4736 there is some evidence favouring the presence of a LLAGN (e.g. the radio source associated with the nucleus of NGC 3627; Filho, Barthel \& Ho 2000) but this is far from overwhelming. However, the similarity in spectral form (when the two reasonably well established accretion-driven systems are excluded) with the predominantly stellar driven systems (including NGC 253), noted in section 5.1, tips the argument against the presence of a dominant LLAGN in these three LINER 2 nuclei. 
Table 6. X-ray flux and luminosity ratios of the LINER 2s.

$\begin{array}{lcccc}\text { Galaxy } & F_{X}(0.5-1) / F_{X}(2-5) & \log L_{X} / L_{B} & \log L_{X} / L_{F I R} & \log L_{X} / L_{H \alpha} \\ & & & & \\ \text { NGC 3627 } & 0.56 & -3.4 & -3.9 & 1.0 \\ \text { NGC 3628 } & 0.18 & -3.3 & -4.4 & 2.8 \\ \text { NGC 4321 } & 0.59 & -3.1 & -4.2 & 1.1 \\ \text { NGC 4569 } & 0.72 & -3.5 & -3.4 & -0.6 \\ \text { NGC 4594 } & 0.38 & -3.4 & -2.8 & 1.2 \\ \text { NGC 4736 } & 0.80 & -3.3 & -3.8 & 1.5 \\ \text { NGC 7217 } & 0.63 & -3.4 & -3.5 & 0.1\end{array}$

The study of this small sample of LINER 2s has clearly advanced the case for heterogeneity in the processes underlying the LINER 2 phenomenon. However, much more definitive analysis is required and indeed will be possible with X-ray measurements which combine good spatial resolution, reasonable spectral resolution and, ideally, better temporal sampling. The search for LLAGN in nearby galaxies of all types is, of course, a high priority for both the $C H A N D R A$ and XMM-NEWTON missions.

\section{ACKNOWLEDGMENTS}

NJS and TPR gratefully acknowledge financial support from PPARC. The archival X-ray data used in this work were all obtained from the Leicester Database and Archive Service (LEDAS) at the Department of Physics \& Astronomy, University of Leicester. The Digitised Sky Survey was produced at the Space Telescope Science Institute, under US government grant NAG W-2166 from the original National Geographic-Palomar Sky Survey plates. We thank the anonymous referee for suggestions that have led to the improvement of this paper.

\section{REFERENCES}

Barth A.J., Ho L.C., Filippenko A.V., Sargent W.L.W., 1998, ApJ, 496, 133

Bregman J.N., Glassgold A.E., 1982, ApJ, 263, 564

Buta R., van Driel W., Braine J., Combes F., Wakamatsu K., Sofue Y., Tomita A., 1995, ApJ, 450, 593

Canizares C.R., Fabbiano G., Trinchieri G., 1987, ApJ, 312, 503

Colbert E.J.M., Mushotzky R.F., 1999, ApJ, 519, 89

Cui W., Feldkhun D., Braun R., 1997, ApJ, 477, 693

Dahlem M., Heckman T.M., Fabbiano G., 1995, ApJ, 442, L49

Dahlem M., Heckman T.M., Fabbiano G., Lehnert M.D., Gilmore D., 1996, ApJ, 461, 724

Dahlem M., Weaver K.A., Heckman T.M., 1998, ApJS, 118, 401

Fabbiano G., 1989, ARA\&A, 27, 87

Fabbiano G., Heckman T., Keel W.C., 1990, ApJ, 355, 442

Fabbiano G., Juda J.Z., ApJ, 1997, 476, 666

Fabbiano G., Kim D.-W., Trinchieri G., 1992, ApJS, 80, 531

Filho M.E., Barthel P.D., Ho L.C., 2000, ApJS, 129, 93

Haynes M.P., Giovanelli R., Roberts M.S., 1979, ApJ, 229, 83

Heckman T.M., 1980, A\&A, 87, 152

Ho L.C., 1999, ApJ, 516, 672

Ho L.C., Filippenko A.V., Sargent W.L.W., 1997, ApJS, 112, 315

Immler S., Pietsch W., Aschenbach B., 1998, A\&A, 331, 601

Irwin J.A., Bregman J.N., 1999, ApJ, 527, 125

Iyomoto N., Makishima K., Matsushita K., Fukazawa Y., Tashiro M., Ohashi T., 1998, ApJ, 503, 168
Ji L., Chen Y., Huang J.H., Gu Q.S., Lei S.J., 2000, A\&A, 355, 922

Junkes N., Hensler G., 1996, in Zimmerman H.U., Trümper J., Yorke H., eds. Proc. Rontgenstrahlung from the Universe, MPE report 263, 459

Keel W.C., 1996, PASP, 108, 917

Kormendy J., 1988, ApJ, 335, 40

Kormendy J., Bender R., Ajhar E.A., Dressler A., Faber S.M., Gebhardt K., Grillmair C., Lauer T.R., Richstone D., Tremaine S., 1996, ApJL, 473, 91

Larkin J.E., Armus L., Knop R.A., Soifer B.T., Matthews K., 1998, ApJS, 114, 59

Lawrence A., 1999, in Schmitt H.R., Kinney A.L., Ho L.C., eds., Adv. in Sp. Res., Vol. 23, No. 5/6, 'The AGN/normal galaxy connection', pg. 1167, Oxford:Elsevier

Makishima K., Kubota A., Mizuno T., Ohnishi T., Tashiro M., Aruga Y., Asai K., Dotani T., Mitsuda K., Ueda Y., Uno S., Yamaoka K., Ebisawa K., Kohmura Y., Okada K., 2000, ApJ, 535,632

Maoz D., Filippenko A.V., Ho L.C., Rix H.-W., Bahcall J.N., Schneider D.P., Macchetto F.D., 1995, ApJ, 440, 91

Maoz D., Koratkar A., Shields J.C., Filippenko A.V., Sternburg A., 1998, AJ, 116, 55

Matsumoto H., Tsuru T.G., Koyama K., Awaki H., Canizares C.R., Kawai N., Matsushita S., Prestwich A., Ward M., Zezas A.L., Kawabe R., 2001, ApJ, in press

Matsushita K., Makishima K., Awaki H., Canizares C.R., Fabian A.C., Fukazawa Y., Loewenstein M., Matsumoto H., Mihara T., Mushotzky R.F., Ohashi T., Ricker G.R., Serlemitsos P.J., Tsuru T., Tsusaka Y., Yamasaki T., 1994, ApJL, 436, 41

Nicholson K.L., Reichert G.A., Mason K.O., Puchnarewicz E.M., Ho L.C., Shields J.C., Filippenko A.V., 1998, MNRAS, 300, 893

Palumbo G.G.C., Maccacaro T., Zamorani G., Panagia N., Vettolani G., 1981, ApJ, 247, 484

Pietsch W., Roberts T.P., Sako M., Freyberg M.J., Read A.M., Borozdin K.N., Branduardi-Raymont G., Cappi M., Ehle M., Ferrando P., Kahn S.M., Ponman T.J., Ptak A., Shirey R.E., Ward M., 2001, A\&A, in press

Pogge R.W., Maoz D., Ho L.C., Eracleus M., 2000, ApJ, 532, 323

Ptak A., Serlemitsos P., Yaqoob T., Mushotzky R., 1999, ApJS, 120,179

Ptak A., Serlemitsos P., Yaqoob T., Mushotzky R., Tsuru T., 1997, AJ, 113, 1286

Roberts T.P., Warwick R.S., 2000, MNRAS, 315, 98

Roberts T.P., Warwick R.S., Ohashi T., 1999, MNRAS, 304, 52

Shields J.C., 1992, ApJL, 399, 27

Smith B.J., Lester D.F., Harvey P.M., Pogge R.W., 1991, ApJ, 373,66

Smith B.J., Harvey P.M., Colomé C., Zhang C.Y., DiFrancesco J., Pogge R.W., 1994, ApJ, 425, 91

Stauffer J.R., Kenney J.D., Young J.S., 1986, AJ, 91, 1286

Taniguchi Y., Wada K., 1996, ApJ, 469, 581

Taniguchi Y., Ohyama Y., Yameda T., Mouti H., Yoshida M., 1996, ApJ, 467, 215 
Terashima Y., Ho L.C., Ptak A.F., Mushotzky R.F., Serlemitsos P.J., Yaqoob T., Kuneida H., 2000a, ApJ, 533, 729

Terashima Y., Ho L.C., Ptak A.F., Yaqoob T., Kuneida H., Kazutami M., Serlemitsos P.J., 2000b, ApJL, 535, 79

Terlevich R., Melnick J., 1985, MNRAS, 213, 841

Turner J.L., Ho P.T.P., 1994, ApJ, 421, 122

Yaqoob T., Serlemitsos P.J., Ptak A., Mushotzky R., Kuneida H., Terashima Y., 1995, ApJ, 455, 508

\section{APPENDIX A: NOTES ON THE SAMPLE GALAXIES}

\section{A1 NGC 3627}

NGC 3627 (M 66) is an interacting galaxy in the Leo triplet. HFS classify it as T2/S2 type, indicating some ambiguity in its optical emission-line ratios leading to doubt as to whether the nucleus is a LINER/H II composite or Seyfert 2-like (with the former being the most probable option). Filho, Barthel \& Ho (2000) report the presence of a variable, flat spectrum radio source in the nucleus of NGC 3627 consistent the presence of a LLAGN.

NGC 3627 was detected with a count rate of $\sim$ 0.1 count s $^{-1}$ in the ROSAT All-Sky Survey, but the only previously reported observation is from a ROSAT PSPC image of NGC 3628 , where NGC 3627 is $36^{\prime}$ off-axis (Dahlem et al 1996). The strong off-axis degradation of the instrument point spread function prevented any useful spatial analysis, but spectral analysis was possible. The data were reasonably well fitted by a combined thermal plasma and power-law continuum model $(k T=0.34 \pm 0.69 \mathrm{keV}, \Gamma=2.0 \pm 0.59$ respectively) with no evidence for absorption above the Galactic line-of-sight value. The inferred $0.1-2.0 \mathrm{keV}$ luminosity was $5 \times 10^{39} \mathrm{erg} \mathrm{s}^{-1}$ for an assumed distance of $6.6 \mathrm{Mpc}$.

\section{A2 NGC 3628}

NGC 3628 is an edge-on galaxy, also in the Leo Triplet, that shows clear morphological distortions (e.g. in H I ; Haynes et al. 1979) due to its interaction with the other Leo Triplet galaxies. Although the nucleus is obscured by a prominent dust lane, there is evidence for a nuclear starburst, though it may be intrinsically less active than in the prototypical starburst galaxies NGC 253 and M 82 (see Dahlem et al. 1996 and references therein).

The presence of the nuclear starburst has made NGC 3628 a popular target for X-ray observations. EINSTEIN observations of the galaxy detected two point X-ray sources, one of which was coincident with the nucleus (Bregman \& Glassgold 1982), together with an X-ray bright outflow aligned along the minor axis of the galaxy (Fabbiano, Heckman \& Keel 1990). The presence of the latter was confirmed by ROSAT PSPC observations (Dahlem et al. 1996). The nuclear source has shown a remarkable level of variability, with its flux changing by factors of up to $\sim 20$ between observations. This establishes the domination of a single source which may be either a LLAGN or luminous X-ray binary (Dahlem, Heckman \& Fabbiano 1995). If it is a luminous binary, then its luminosity is such that it is probably an example of the so-called ultra-luminous X-ray source phenomena (ULX; Makishima et al. 2000) situated close to the dynamical centre of the galaxy, as is seen in many nearby galax- ies (Colbert \& Mushotzky 1999). Spectral studies of NGC 3628 using ASCA have shown the nuclear source to have a very flat spectral shape, with simple powerlaw fits giving a spectral index $\Gamma \approx 1.2$ (Yaqoob et al. 1995). More complicated models (including one or more thermal plasma components and a heavily-absorbed power-law continuum) have also been successfully fitted to the $A S C A$ and ROSAT PSPC data (Dahlem, Weaver \& Heckman 1998; Ptak et al. 1999).

\section{A3 NGC 4321}

A remarkable feature of the well-known grand design spiral NGC 4321 (M 100) is the number of recent supernovae in the galaxy, numbering 4 in the last 100 years. The $E I N$ STEIN observatory targeted the most recent, SN1979C, two years after its appearance, but it did not detected the SN in X-rays (Palumbo et al. 1981). Instead, EINSTEIN saw a bright nuclear source and a second source in the northern spiral arm. Immler, Pietsch \& Aschenbach (1998) report the results of a ROSAT HRI observation of NGC 4321 which detects several point sources within the $\mathrm{D}_{25}$ ellipse of the galaxy, including the bright nuclear source (which has a luminosity of $\mathrm{Lx}_{\mathrm{X}} \approx 6 \times 10^{39} \mathrm{erg} \mathrm{s}^{-1}$ ) and SN 1979C (at a luminosity of $\mathrm{L}_{\mathrm{X}}=1 \times 10^{39} \mathrm{erg} \mathrm{s}^{-1}$ ), 16 years after its initial outburst. NGC 4321 has not been studied spectroscopically in X-rays to date.

\section{A4 NGC 4569}

NGC 4569 (M 90) is in the Virgo cluster. It has a very bright nucleus, probably the result of a recent star formation episode (Stauffer, Kenney \& Young 1996). Optical and IUE studies (Keel 1996) have demonstrated that the nucleus is the host to a young star cluster, dominated optically by A-type supergiants. HST UV imaging showed the nucleus to be a bright, point-like, UV source (Maoz et al. 1995), although more recent observations indicate that the UV source is clearly extended (Barth et al. 1998). UV spectroscopy reveals that the dominant continuum source is a cluster of massive stars and that there is no evidence in the UV for a LLAGN (Maoz et al. 1998). Narrow-band ([O III ] $\lambda 5007$ and $\mathrm{H} \alpha+[\mathrm{N} \mathrm{II}]) H S T$ optical imaging on the other hand, shows the nucleus to be bright and unresolved on a sub-arcsecond scale (Pogge et al. 2000).

A ROSAT PSPC observation reveals extended X-ray emission in the bulge and disk of NGC 4569, with the emission peaking at the nucleus (Junkes \& Hensler 1996). The HRI observation, however, shows a luminous, unresolved point source coincident with the nucleus (Colbert \& Mushotzky 1999; Roberts \& Warwick 2000). ASCA observations of NGC 4569 confirm that the X-ray emission is extended on scales of several $\mathrm{kpc}$ in both the soft $(0.5-2 \mathrm{keV})$ and hard (2- $7 \mathrm{keV}) A S C A$ bands (Terashima et al. 2000a). The $A S C A$ spectra are best described by a Raymond-Smith plus power-law (or thermal bremsstrahlung) model, with parameters $k T \approx 0.67$ and $\Gamma \approx 2.2$ respectively; an additional column density of $1.7 \times 10^{21}$ atoms $\mathrm{cm}^{-2}$ applies to the second component. Terashima et al. (2000a) postulate that there is no evidence for the presence of an AGN in NGC 4569; the soft component could originate in star formation, whereas the hard component could easily be the sum of a discrete (non-AGN) source contribution. In combination with 
the other measurements summarised above, Terashima et al. (2000a) conclude that the LINER spectrum of NGC 4569 is the probably the result of the presence of hot stars in its nucleus.

\section{A5 NGC 4594}

The famous galaxy NGC 4594 (M 104, the Sombrero) was one of the earliest galaxies to show evidence for the possible presence of a super-massive (up to $10^{9} M_{\odot}$ ) black hole in its nucleus (Kormendy 1988). It was classified by HFS as having a LINER 2 nucleus, and there is an abundance of evidence to suggest that it is powered by a LLAGN. Most convincingly, the high-resolution HST spectrum of NGC 4594 shows broad wings on the nuclear emission lines (Kormendy et al. 1996).Examination of the ionization budget for the optical lines highlights a deficit of near-UV ionizing photons which points to a non-stellar component in the nucleus (Maoz et al. 1998), as does the FIR-radio ratio when compared to Wolf-Rayet galaxies (Ji et al. 2000). Intriguingly, the SED of the nucleus does not show evidence for a "big blue bump" as seen in more luminous AGN, although this may be an implicit characteristic of the LLAGN class (Ho 1999).

The X-ray emission of NGC 4594 has been very well studied. EINSTEIN showed an extended X-ray source to be centred on the nucleus of the galaxy (Fabbiano, Kim \& Trinchieri 1992). Fabbiano \& Juda (1997) investigated a ROSAT HRI observation, finding that the emission broke down into three components: a bright, point-like nuclear source with $\mathrm{L}_{\mathrm{X}} \sim 3.5 \times 10^{40} \mathrm{erg} \mathrm{s}^{-1}(0.1-2.4 \mathrm{keV})$, clumpy disk emission and unresolved bulge component. They concluded that if the nuclear emission is due to accretion onto a super-massive black hole, then it must be severely subEddington. $A S C A$ spectroscopy was investigated by Nicholson et al. (1998); the best fit to the combined $A S C A$ and $R O S A T$ PSPC data is a power-law continuum with $\Gamma \approx 1.6$ and $N_{H}$ slightly above the Galactic value. However, if a putative soft X-ray cut-off is masked by the extended thermal emission component then the absorption on the power-law component could rise to $3 \times 10^{21}$ atoms $\mathrm{cm}^{-2}$. Nicholson et al. also look at $H S T$ UV spectra, and find the properties of NGC 4594 are consistent with the presence of a LLAGN. Ptak et al. (1999) also fit a 2-component model to the $A S C A$ and PSPC data, with similar results except that they find more intrinsic absorption on the powerlaw $\left(\sim 10^{22}\right.$ atoms $\left.\mathrm{cm}^{-2}\right)$.

\section{A6 NGC 4736}

NGC 4736 (M 94) hosts the closest example of a LINER 2 nucleus, and so is amongst the best studied. There is conflicting evidence as to whether the LINER 2 in NGC 4736 is powered by a LLAGN. Larkin et al. (1998) conducted a near-infrared spectroscopic survey of LINER galaxies and found NGC 4736 to have the largest $[\mathrm{Fe} \mathrm{II}] / \mathrm{Pa} \beta$ ratio in their sample, indicating that its excitation was dominated by stellar processes. Both the $\mathrm{H} \alpha$ luminosity (Taniguchi et al. 1996) and FIR emission (Smith et al. 1991; Smith et al. 1994) may also be explained solely by stellar emission. On the other hand, Turner \& Ho (1994) detect a strong nonthermal radio continuum source at the position of the nucleus. Maoz et al. (1995) image the nucleus in UV using the
$H S T$, and detect two bright point sources in the nuclear region and possible bow shocks, leading to the conjecture that two objects are in the final stages of merging in the nucleus. One or both of these sources may be very compact star clusters, but they may also be super-massive black holes, one of which comes from a merged, nucleated satellite galaxy (as postulated in Taniguchi \& Wada 1996).

Cui et al. (1997) studied a ROSAT PSPC observation of NGC 4736 which revealed a bright, nuclear X-ray source surrounded by an extended region of soft $(k T \approx 0.3 \mathrm{keV})$ thermal gas. The PSPC data was re-analysed, along with two ROSAT HRI observations and an ASCA observation of the galaxy, by Roberts, Warwick \& Ohashi (1999). They found the X-ray spectrum to be dominated by a Seyfert-like power-law continuum with slope $\Gamma \approx 1.7$, with little intrinsic absorption. Below $2 \mathrm{keV}$ two thermal emission components were required with $k T$ in the range $0.1-0.6 \mathrm{keV}$. The possible detection of an Fe $\mathrm{K} \alpha$ feature at $6.8 \mathrm{keV}$ was also reported. The compact nuclear source was found to have a luminosity of $6 \times 10^{39} \mathrm{erg} \mathrm{s}^{-1}(0.5-10 \mathrm{keV})$ and thus may be a near-quiescent AGN. However, the alternative hypothesis that the hard component originates entirely in X-ray binaries could not be ruled out.

\section{A7 NGC 7217}

NGC 7217 is one of the most spheroid-dominated spiral galaxies known, containing a series of ring structures (Buta et al. 1995). Most notable of these is a central dust ring, $\sim 17^{\prime \prime}$ in diameter, around a dust-free nucleus. No UV emission was detected from the nucleus by Barth et al. (1998). The NIR spectroscopic survey of Larkin et al. (1998) found the nucleus to have a high $[\mathrm{Fe} \mathrm{II}] / \mathrm{Pa} \beta$ ratio, and therefore to be dominated by stellar processes, a conclusion which is backed up by the FIR-radio relation of Ji et al. (2000).This galaxy has not been previously studied in X-rays. 
This figure "figure1.gif" is available in "gif" format from: http://arxiv.org/ps/astro-ph/0101475v1 